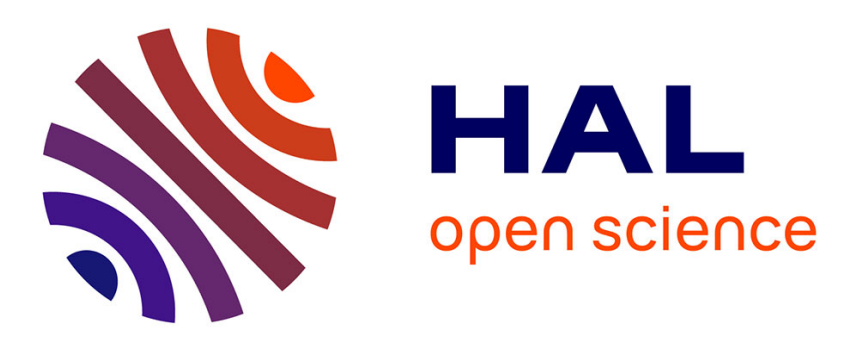

\title{
Programmable metamaterials with digital synthetic impedance circuits for vibration control
}

Kaijun Yi, Gaël Matten, Morvan Ouisse, Emeline Sadoulet-Reboul, Manuel

Collet, Gaël Chevallier

\section{- To cite this version:}

Kaijun Yi, Gaël Matten, Morvan Ouisse, Emeline Sadoulet-Reboul, Manuel Collet, et al.. Programmable metamaterials with digital synthetic impedance circuits for vibration control. Smart Materials and Structures, 2020, 29 (3), pp.035005. 10.1088/1361-665X/ab6693 . hal-03026157

\section{HAL Id: hal-03026157 https://hal.science/hal-03026157}

Submitted on 26 Nov 2020

HAL is a multi-disciplinary open access archive for the deposit and dissemination of scientific research documents, whether they are published or not. The documents may come from teaching and research institutions in France or abroad, or from public or private research centers.
L'archive ouverte pluridisciplinaire HAL, est destinée au dépôt et à la diffusion de documents scientifiques de niveau recherche, publiés ou non, émanant des établissements d'enseignement et de recherche français ou étrangers, des laboratoires publics ou privés. 


\title{
Programmable metamaterials with digital synthetic impedance circuits for vibration control
}

\author{
Kaijun $\mathrm{Yi}^{\mathrm{a}, \mathrm{b}, *}$, Gaël Matten ${ }^{\mathrm{b}}$, Morvan Ouisse ${ }^{\mathrm{b}}$, Emeline Sadoulet-Reboul ${ }^{\mathrm{b}}$, Manuel \\ Collet $^{\mathrm{c}}$, Gaël Chevallier ${ }^{\mathrm{b}}$ \\ ${ }^{a}$ School of Aerospace Engineering, Beijing Institute of Technology, Beijing 100081, PR China \\ ${ }^{b}$ Univ. Bourgogne Franch-Comté, FEMTO-ST Institute, CNRS/UFC/ENSMM/UTBM, Department of \\ Applied Mechanics, 24 rue de l'épitaphe, 25000 Besançon, France \\ ${ }^{c}$ Univ Lyon, Ecole Centrale de Lyon, ENISE, ENTPE, CNRS, Laboratoire de Tribologie et Dynamique \\ des Systèmes LTDS UMR5513, F-69134 Ecully, France
}

\begin{abstract}
This paper studies a new type of smart metamaterial consisting of piezoelectric actuators and digital synthetic impedance circuits (digital circuits). The digital circuit contains a micro-controller. By programming the micro-controller, the circuit can establish any desired impedance between the terminals of the connected piezoelectric patch, therefore to program the dynamical behaviors of the designed metamaterial. In this sense, a programmable metamaterial is studied. Experiments are conducted to study its programmable dynamical behaviors, with a particular focus on vibration reduction. Numerical simulations are also done in this work to qualitatively verify the experimental results. Different design methods are used to customize the dynamical behaviors of the designed metamaterial. Results demonstrate that the proposed smart metamaterial can be flexibly programmed online to reduce vibration at desired frequencies or within a large frequency band. The proposed programmable metamaterial conception can be naturally extended to other kinds of structures like plates and shells, they are promising components in the future adaptive structures.
\end{abstract}

Keywords: piezoelectric material, metamaterials, band gap, vibration control, synthetic impedance

\section{Introduction}

The intensive use of light-weight structures in modern aerospace and transportation industries results in more serious vibration and noise issues due to their high dynamical sensitivities to disturbance. In recent years, the emerging metamaterials provide new ways to deal with these issues thanks to their unprecedented wave manipulation abilities. Recently, in order to design metamaterials with tunable vibro-acoustic properties therefore to make the structures be adaptive to different working conditions and environments, piezoelectric materials have been integrated into structures, results in so-called

\footnotetext{
${ }^{*}$ Corresponding author

Email address: kaijun.yi@bit.edu.cn (Kaijun Yi)
} 
smart metamaterials [1]. Efforts have been dedicated to design smart metamaterials that can be used for vibration[2] and noise control[3] or wave propagation manipulation[4]. In many of these studies, it has been demonstrated that the vibro-acoustic behavior of smart metamaterials can be controlled through external electrical shunts. However, there is still a lack of studies relate to the programmable (smart) metamaterial, whose dynamical behaviors can be online programmed, especially when it comes to the application of vibration reduction. The presented paper is related in particular to this topic, it contributes towards the design and analysis of programmable metamaterials capable of reducing structural vibration at desired frequencies or within a desired wide frequency band.

Metamaterials for acoustic waves were first proposed by Liu and his collaborators [5]. Later, piezoelectric materials with shunting circuits have been introduced into the design of metamaterials in order to obtain smart materials with tunable properties. Piezoelectric patches shunted with inductance and resistance have been first proposed by Forward [6] to damp mechanical vibrations. The inductance and the inherent capacitance of the patch naturally introduce a resonator into the system. The resonance frequency can be tuned by changing the value of inductance [7]. Patches shunted with resonant circuits are periodically placed along rods to control the longitudinal wave propagation in Thorp et al.'s work [8]. Tunable band gaps generated by using shunted piezoelectric materials are demonstrated. Following Thorp et al.'s work, patches shunted with resonant circuits are proposed to control flexural waves in beams $[9,10]$ and plates $[11]$ for the purpose of vibration and noise control. The mechanism behind the creation of band gaps caused by resonant shunts is not clear until the work of Airoldi and Ruzzene [12] was published. They demonstrate that the resonant behavior of piezo-patches with resonant shunts is reflected in the mechanical behavior of the equivalent bending and axial stiffness of the studied metamaterial beam. The band gaps are related to the resonant behavior of the equivalent stiffness. From the perspective of material, these results indicate that the dynamical response of the metamaterial can be changed by tuning the equivalent material parameters of it through the external circuits. Except resonant shunts, negative capacitance circuits are also widely used in designing smart metamaterials. The negative capacitance is also first proposed by Forward [13]. It can cancel a part of the inherent capacitance of the patch to significantly enhance the electro-mechanical coupling effect. Therefore, it is widely used for vibration reduction $[14,15]$. Recent researches show that negative capacitance can change the effective static stiffness of the system. This property has been explored to enlarge band gaps [16, 17] or manipulate wave propagation $[18,19]$ in metamaterials. In a word, plenty of works have demonstrated that piezoelectric patches with shunts can be used to change the equivalent material parameters therefore to tune the dynamical responses of the structures. However, in the previous studies shunting circuits are commonly made by analog elements, this fact makes the metamaterials with shunting circuits are difficult to be tuned in real-time, more advanced smart metamaterials need to be proposed.

One promising trend in designing smart metamaterials is using synthetic impedance circuit. This kind of circuit can establish a desired impedance between the voltage on the terminal of a patch and the current flowing out the same patch. A digital signal processor (DSP) is commonly used in the circuit to implement the designed control law, which is 
the transfer function of the desired impedance. To realize such circuit in practice, one can measure the current or charge flowing out from the patch, then feed voltage according to the control law back to the patch [20, 21]. However, the hysteresis effect between the voltage and deformation inside the piezo-materials degenerates the control effect especially when the driving voltage is large [22]. Therefore, it is recommended to measure the voltage and drive the patch through current or charge. Fleming et al. [23, 24] first proposed to use the synthetic impedance to realize piezoelectric shunt-damping. Matten et al. [25] and Nečásek et al. [26] recently also independently developed synthetic impedance circuits for vibration control. Synthetic impedance circuits can be controlled in real-time, also it inherits the simplicity of shunting techniques. Therefore, they are promising for new types of programmable metamaterials. Sugino et al. [27] studied a piezoelectric metamaterial beam with synthetic impedance circuits. Their numerical results show that the piezometamaterial beam could be designed to behave in completely different ways by using different control law. However, no experimental results are presented in their work.

In this paper, a smart metamaterial beam using synthetic impedance circuits is designed, experiments are conducted to study its programmable dynamical behaviors, with a particular focus on vibration reduction. Numerical simulations are also done in this work to qualitatively verify the experimental results. The rest of this paper is organized as follows: Sec. 2 introduces the numerical tools, the synthetic impedance circuit and experimental setup. In detail, the numerical model of the programmable metamaterial is presented in Sec. 2.1. The wave finite element method (WFEM) used to analyze the dispersion relations of the waves is recalled in Sec. 2.2. The synthetic impedance circuit and experimental setup are introduced in Secs 2.3 and 2.4, respectively. Sec. 3 discusses the numerical and experimental results. The preliminary results prepared for the following discussions are presented in Sec. 3.1. The programmable dynamical responses of the designed metamaterial in terms of vibration reduction are analyzed in Sec. 3.2. Sec. 4 concludes the whole paper.

\section{Numerical tools, synthetic impedance circuit and experimental setup}

\subsection{Numerical model of the programmable metamaterial}

Figure 1(a) shows the schematic of the studied programmable metamaterial. The beam is composed of 10 and a half cells. The host beam in each unit cell contains two segments with different thicknesses. The patches for control are glued on the thin segment. The patches are connected with a synthetic impedance circuit in each cell, which establishes an admittance $G_{i}$ on them through the law $I_{c, i}=G_{i} V_{c, i}$, in which i represents the number of the cell, $I_{c, i}$ and $V_{c, i}$ are the current flowing out from the patch and the voltage on the electrode of the patch, respectively. The structure is excited by the patches in the half cell (cell 0) with applied voltage $V_{e}$. The acceleration along $\mathrm{z}$ axis at the red point near the left end is studied for discussion. Figure 1(b) shows the manufactured beam with glued

patches. The geometry parameters of the patches and the measured mean dimensions of the host beam in one unit cell are listed in Table 1.

Numerical simulations are done using the finite element method (FEM), their results will be used as references during the discussion. The FE-form governing equations of the 


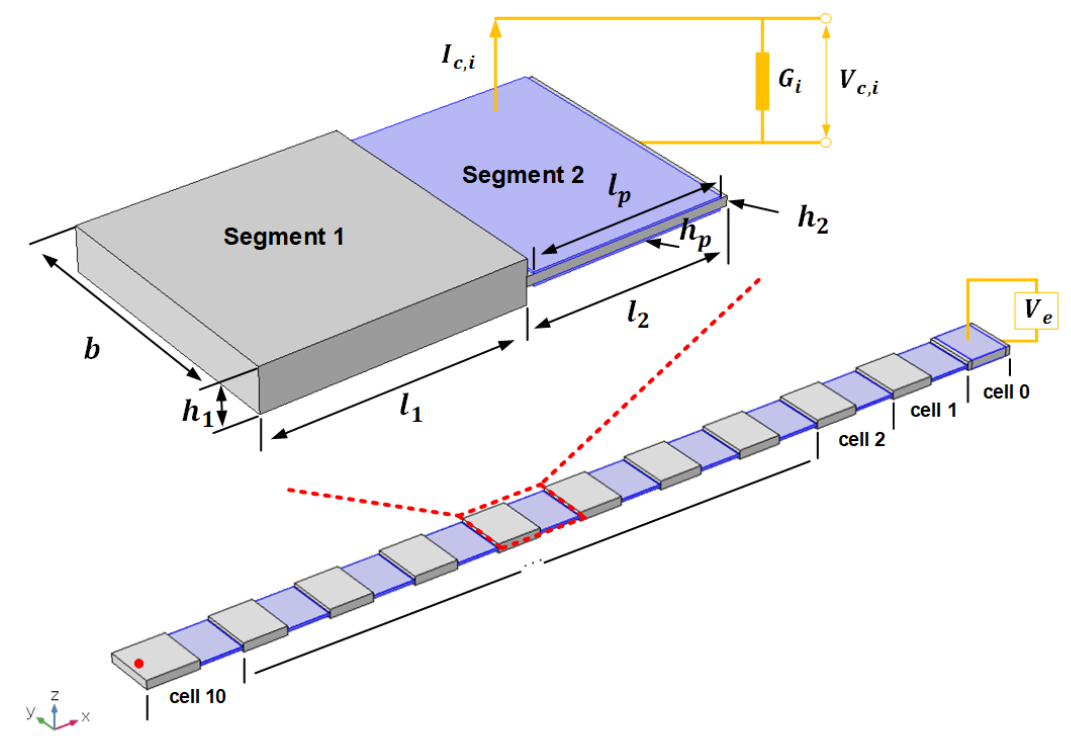

(a)

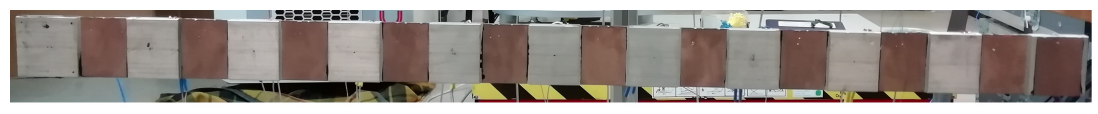

(b)

Figure 1: (a) Schematic of the programmable metamaterial. $G_{i}$ is the admittance applied on the patches in cell i, $I_{c, i}=G_{i} V_{c, i}$. The structure is excited by the patches on the right end, $V_{e}$ is the excitation voltage. The acceleration along $\mathrm{z}$ axis at the red point near the left end is studied. (b) The fabricated beam with glued patches.

programmable metamaterial are

$$
\begin{gathered}
\mathbf{M} \ddot{\boldsymbol{d}}+\mathbf{C}_{d} \dot{\boldsymbol{d}}+\mathbf{K} \boldsymbol{d}+\left[\mathbf{H}_{d v e} \mathbf{H}_{d v c}\right]\left[\begin{array}{c}
\boldsymbol{V}_{e} \\
\boldsymbol{V}_{c}
\end{array}\right]=\boldsymbol{f} \\
-\left[\begin{array}{c}
\mathbf{H}_{d v e}^{T} \\
\mathbf{H}_{d v c}^{T}
\end{array}\right] \dot{\boldsymbol{d}}+\left[\begin{array}{cc}
\mathbf{C}_{p e} & 0 \\
0 & \mathbf{C}_{p c}
\end{array}\right]\left[\begin{array}{c}
\dot{\boldsymbol{V}}_{e} \\
\dot{\boldsymbol{V}}_{c}
\end{array}\right]=-\left[\begin{array}{c}
\boldsymbol{I}_{e} \\
\boldsymbol{I}_{c}
\end{array}\right]
\end{gathered}
$$

in which, $\boldsymbol{d}$ is the mechanical displacement vector, $\boldsymbol{V}_{e}$ and $\boldsymbol{V}_{c}$ represent voltages of the patches for excitations and voltages on the patches for control, respectively; $\mathbf{M}, \mathbf{C}_{d}$ and $\mathbf{K}$ are the mass matrix, damping matrix and stiffness matrix, respectively; $\mathbf{H}_{d v}=\left[\mathbf{H}_{d v e} \mathbf{H}_{d v c}\right]$ is the coupling matrix, and $\mathbf{C}_{p e}, \mathbf{C}_{p c}$ are intrinsic capacitances of the patches; lastly, $\boldsymbol{f}$ represents the mechanical excitation, which is zero in our simulations, $\boldsymbol{I}_{e}$ and $\boldsymbol{I}_{c}$ are the currents flowing outside the patches.

The control is introduced into the system through the following boundary condition

$$
\boldsymbol{I}_{c}=\mathbf{G}(j \omega) \boldsymbol{V}_{c},
$$

in which, $j=\sqrt{-1}$ and $\omega$ is the circular frequency, recall that $\mathbf{G}=\operatorname{diag}\left(G_{i}\right)$ is the control law used to establish required admittance between the electrodes of the patches, the specific expression of it will be given during the discussion of the results. 
Table 1: Geometrical parameters of the manufactured beam and the patches.

\begin{tabular}{lll}
\hline Parameter & Variable [unit] & Segment 1 \\
\hline Length of the patch & $l_{p}[\mathrm{~mm}]$ & 45 \\
Thickness of the patch & $h_{p}[\mathrm{~mm}]$ & 0.5 \\
Width & $b[\mathrm{~mm}]$ & 60 \\
Mean length of segment 1 & $l_{1}[\mathrm{~mm}]$ & 52.1 \\
Mean length of segment 2 & $l_{2}[\mathrm{~mm}]$ & 49.3 \\
Mean thickness of segment 1 & $h_{1}[\mathrm{~mm}]$ & 10.1 \\
Mean thickness of segment 2 & $h_{2}[\mathrm{~mm}]$ & 2.2 \\
Total length of the beam & $l_{\text {tot }}[\mathrm{mm}]$ & 1065.3 \\
\hline
\end{tabular}

Assume that the excitation is harmonic, namely, in the form $\boldsymbol{V}_{e} e^{j \omega t}$. Therefore, the governing equations are transfered into the frequency domain, as shown in Eq. (3).

$$
\begin{array}{r}
\left(-\omega^{2} \mathbf{M}+j \omega \mathbf{C}_{d}+\mathbf{K}\right) \boldsymbol{d}+\left[\mathbf{H}_{d v e} \mathbf{H}_{d v c}\right]\left[\begin{array}{l}
\boldsymbol{V}_{e} \\
\boldsymbol{V}_{c}
\end{array}\right]=0, \\
-\left[\begin{array}{c}
\mathbf{H}_{d v e}^{T} \\
\mathbf{H}_{d v c}^{T}
\end{array}\right] \boldsymbol{d}+\left[\begin{array}{cc}
\mathbf{C}_{e e} & 0 \\
0 & \mathbf{C}_{c c}
\end{array}\right]\left[\begin{array}{l}
\boldsymbol{V}_{e} \\
\boldsymbol{V}_{c}
\end{array}\right]=-\frac{1}{j \omega}\left[\begin{array}{l}
\boldsymbol{I}_{e} \\
\boldsymbol{I}_{c}
\end{array}\right] .
\end{array}
$$

Using Eqs. (2) and (3) one can obtain the dynamic responses of the system corresponding to different control laws.

\subsection{Dispersion relation analysis}

The WFEM is used to study the dispersion relations in the 1D programmable metamaterial. Note that, in 2D cases involving piezoelectric materials and shunts, the method proposed in [28] maybe a better choice to study the waves. In the WFEM, wave propagation characteristics can be analyzed using only a single unit cell thanks to the Floquet periodic boundary condition [16]. The symmetrical unit cell illustrated in Fig. 2 is employed. In the absence of any external load, the dynamic equations governing the unit cell are

$$
\mathbf{M}^{c}\left[\begin{array}{c}
\ddot{\boldsymbol{d}}_{L} \\
\ddot{\boldsymbol{d}}_{R} \\
\ddot{\boldsymbol{d}}_{I} \\
\ddot{V}
\end{array}\right]+\mathbf{C}_{d}^{c}\left[\begin{array}{c}
\dot{\boldsymbol{d}}_{L} \\
\dot{\boldsymbol{d}}_{R} \\
\dot{\boldsymbol{d}}_{I} \\
\dot{V}
\end{array}\right]+\mathbf{K}^{c}\left[\begin{array}{c}
\boldsymbol{d}_{L} \\
\boldsymbol{d}_{R} \\
\boldsymbol{d}_{I} \\
V
\end{array}\right]=\left[\begin{array}{c}
\boldsymbol{f}_{L} \\
\boldsymbol{f}_{R} \\
0 \\
0
\end{array}\right],
$$

in which, symbols with subscript $L, R, I$ respectively denote the left-side, right-side and internal mechanical degrees of freedom (DOFs) or force as illustrated in Fig. 2.

Matrices in Eq. (4) can be obtained using any existing FEM package. The control law in Eq. (2) is included in the stiffness matrix in frequency domain

$$
\mathbf{K}^{c}=\left[\begin{array}{cccc}
\mathbf{K}_{L L} & \mathbf{K}_{L R} & \mathbf{K}_{L I} & \mathbf{H}_{L V} \\
\mathbf{K}_{L R}^{T} & \mathbf{K}_{R R} & \mathbf{K}_{R I} & \mathbf{H}_{R V} \\
\mathbf{K}_{L I}^{T} & \mathbf{K}_{R I}^{T} & \mathbf{K}_{I I} & \mathbf{H}_{I V} \\
-\mathbf{H}_{L V}^{T} & -\mathbf{H}_{R V}^{T} & -\mathbf{H}_{I V}^{T} & C_{p}-\frac{G}{j \omega}
\end{array}\right] .
$$


Recall that $\mathbf{H}_{L V}$ and the other similar matrices in Eq. (5) are the coupling terms, $C_{p}$ represents the intrinsic capacitance of the patch.

The Floquet periodic boundary condition requires that displacements on the left and right sides should satisfy

$$
\boldsymbol{d}_{R}=\lambda \boldsymbol{d}_{L}
$$

in which, $\lambda=e^{-j k \Delta}$ describes the amplitude and phase changes when waves propagate from the left end of the unit cell to the right end. $\Delta=l_{1}+l_{2}$ is the length of the cell. $k$ represents the wavenumber, imaginary part of it is the phase constant and the real part is the attenuation factor. Additionally, the force on the two sides should satisfy

$$
\boldsymbol{f}_{R}=-\lambda \boldsymbol{f}_{L}
$$

The aim of dispersion analysis is to find the relationship between the wavenumber $k$ and frequency $\omega$. In the present paper, a $\omega$ is fixed to search for $k$. To do so, first the inner DOFs and the voltage DOF are condensed to obtain

$$
\left[\begin{array}{ll}
\mathbf{D}_{L L} & \mathbf{D}_{L R} \\
\mathbf{D}_{R L} & \mathbf{D}_{R R}
\end{array}\right]\left[\begin{array}{l}
\boldsymbol{d}_{L} \\
\boldsymbol{d}_{R}
\end{array}\right]=\left[\begin{array}{l}
\boldsymbol{f}_{L} \\
\boldsymbol{f}_{R}
\end{array}\right]
$$

in which, $\mathbf{D}=-\omega^{2} \mathbf{M}^{c}+j \omega \mathbf{C}_{d}^{c}+\mathbf{K}^{c}$ is the condensed dynamic stiffness matrix, details of it can be found in $[16,29]$.

Then, substituting Eqs. (6) and (7) into Eq. (8) and eliminating the force vectors, one can have the final eigenvalue problem

$$
\left(\left[\begin{array}{cc}
\mathbf{0} & \mathbf{I} \\
-\mathbf{D}_{R L} & -\mathbf{D}_{R R}
\end{array}\right]-\lambda\left[\begin{array}{cc}
\mathbf{I} & \mathbf{0} \\
\mathbf{D}_{L L} & \mathbf{D}_{L R}
\end{array}\right]\right)\left[\begin{array}{l}
\boldsymbol{d}_{L} \\
\boldsymbol{d}_{R}
\end{array}\right]=0
$$

Solving Eq. (9) at different frequencies and using $k=j \frac{\ln \lambda}{\Delta}$ give the dispersion curves.

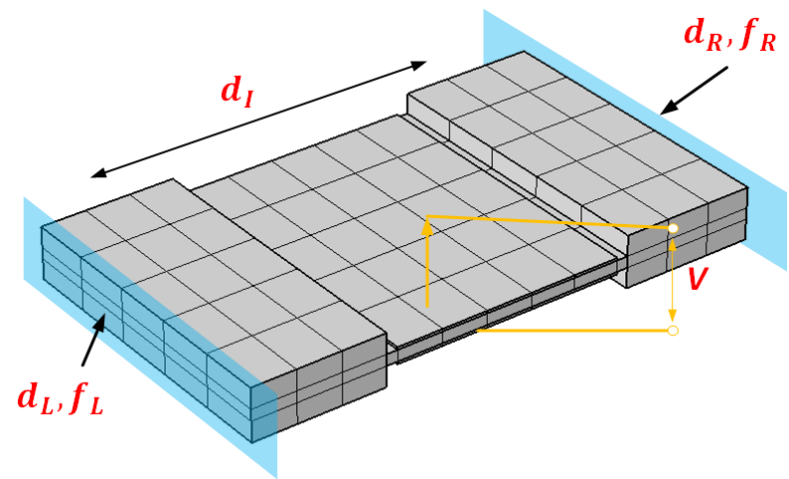

Figure 2: The unit cell used to study the dispersion relations. 


\subsection{Synthetic impedance circuit}

The piezoelectric patches are controlled by digital synthetic impedance circuits (digital circuits for short hereinafter) proposed by G. Matten et al. in their work [25]. Figure 3(a) shows the global architecture of the digital circuit. A micro-controller is used in the circuit, the designed control law is implemented in it. The voltage on the patch is measured using the integrated analog to digital converter (ADC) inside the micro-controller. Before the voltage signal goes into the ADC, the amplitude level of it is adapted to be within the allowable range of the ADC, then the high frequency component of the signal is filtered to avoid aliasing effect. The micro-controller output a voltage through the integrated digital to analog converter (DAC) according to the input signal and the implemented control law. The output voltage is adapted another time, then it is applied on a voltage-driving current source to feed current back to the patch. A real circuit is shown in Fig. 3(b). More details on the architecture of the circuit can be found in [25].

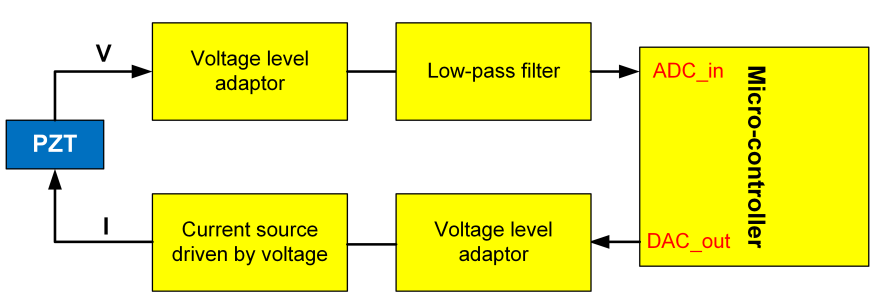

(a)

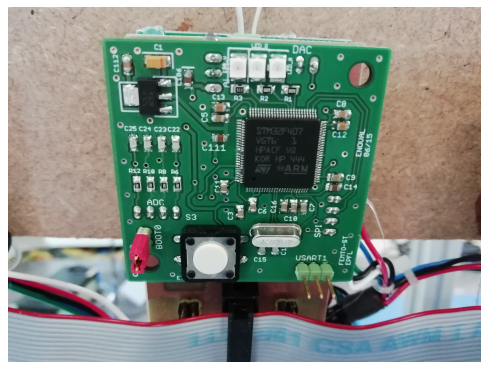

(b)

Figure 3: (a) The global architecture of the digital circuit. (b) The digital circuit.

The computation is done inside the micro-controller in real-time. The control law is discretized in time and is mathematically implemented by a so called infinite impulse response (IIR) filter (see Eq. 10). In this equation, $p$ is the feed-forward filter order, $q$ is the feedback filter order, $a_{j}$ are the feedback filter coefficients, $b_{i}$ are the feed-forward filter coefficients, $x_{k}$ is the input signal and $y_{k}$ is the output signal.

$$
y_{k}=\frac{1}{a_{0}}\left[\sum_{i=0}^{p} b_{i} \cdot x_{k-i}-\sum_{j=1}^{q} a_{j} \cdot y_{k-j}\right] \text {. }
$$

In the experiments, the sampling frequency of the digital micro-controller is $150 \mathrm{kHz}$, which is large enough compared with the maximum interested frequency in this paper.

\subsection{Experimental setup}

Figure 4 shows the experimental setup. The beam is handed up on the ceiling through two soft lines in order to simulate a free-free boundary condition. The beam is made of aluminum and the patches are made of PIC 151, whose material parameters are given in Appendix A. Recall that except the patches for excitation, there are 10 pairs of patches on the beam, only 9 of them are used, as shown in Fig. 4. The 10th patch set is left short-circuited (SC). Each of the 9 patch sets is connected with a digital circuit to form 
a controller. Numbers of these controllers are shown in Fig. 4. The digital circuits are powered by two DC power suppliers, one provide $\pm 30 \mathrm{~V}$ for the op-amplifiers in the circuits and the other one provides $\pm 5 V$ for the micro-processors. A PC is used along with a data acquisition system to generate chirp signals to stimulate the beam and to analyze the data acquired by the accelerometer. The excitation signal is amplified by a factor of 20 using a power amplifier before it is applied on the patches for excitation. The frequency is swept from $100 \mathrm{~Hz}$ to $2000 \mathrm{~Hz}$. The accelerometer has three channels, which provide the acceleration in $\mathrm{x}, \mathrm{y}$ and $\mathrm{z}$ directions, respectively. The transfer function between the excitation voltage $V$ and the measured acceleration along z direction $a_{z}$ will be studied in Sec. 3. The PC is also used to program the digital circuits through a communication line.

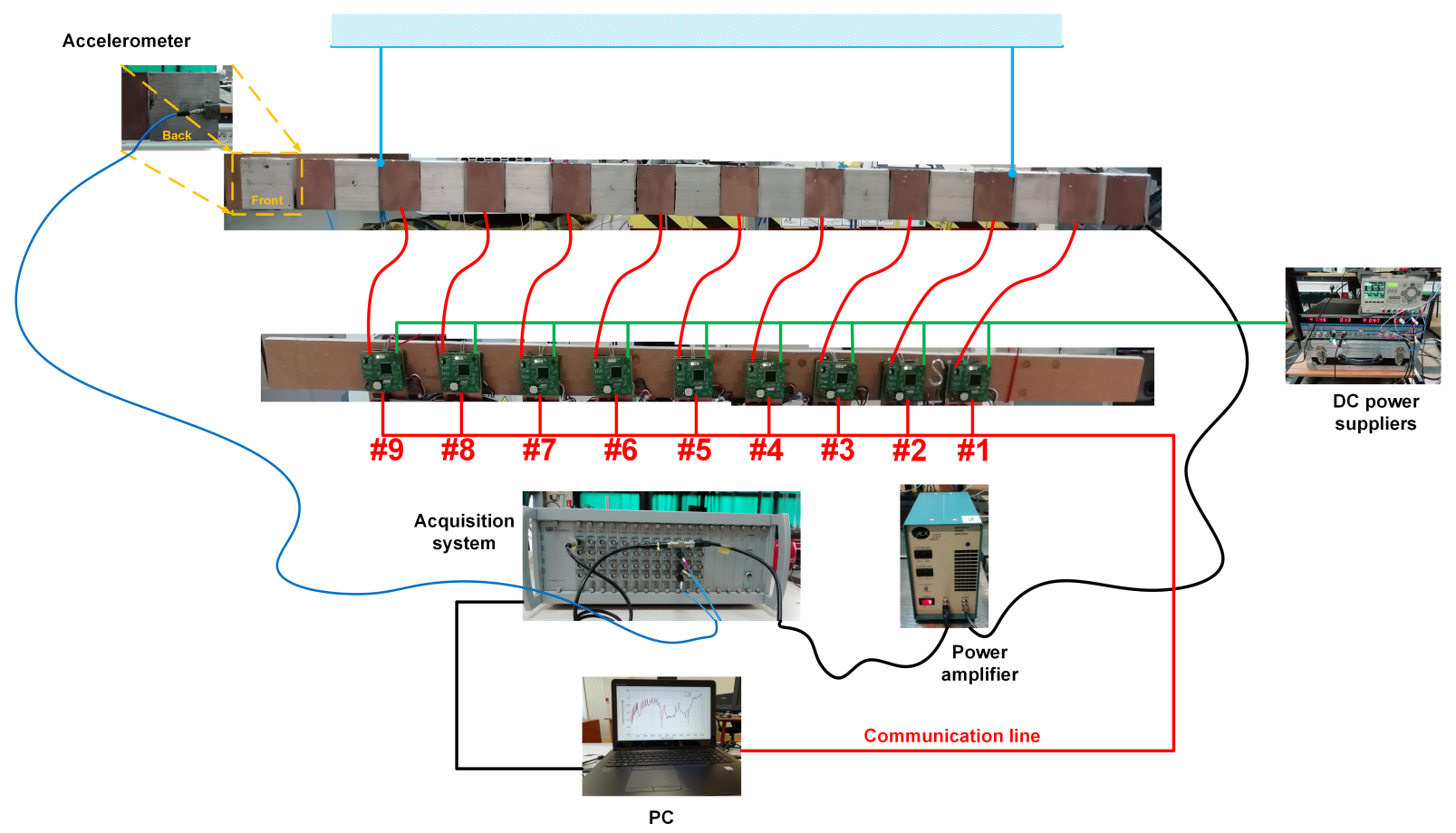

Figure 4: Experimental setup.

\section{Results and discussions}

\subsection{Preliminary}

Numerical simulations and experiments are done to study the programmable dynamic behaviors of the designed metamaterial beam. The numerical results are used as benchmarks to qualitatively verify the experimental results. In this work, the digital circuits are used to simulate resonant shunts, therefore to online control the equivalent bending stiffness and damping of the studied metamaterial beam [12]. The control law used in the digital circuit is

$$
G(s)=\frac{1}{L s+R}
$$


in which, $s$ is the Laplace domain variable. $L$ and $R$ respectively correspond to the inductance and resistance in a typical analog resonant shunt, they are the design parameters. The resonance frequency of the simulated resonant circuit is

$$
\omega_{L C}=\frac{1}{\sqrt{L C_{p}}} .
$$

To study the programmable performances, the metamaterial beam will be programmed to reduce the vibration of desired modes. The vibration reduction is basically realized using band gaps created by resonant behaviors of the digital circuits. The band gap occurs near the vicinity of the resonance frequency expressed in Eq. (12). Therefore, to target a specific mode, the resonance frequency should be designed near the mode by choosing a suitable $L$ value. In the presented work, the $L$ value for a targeted mode is determined by first finding an initial value according to Eq. (12), then manually tuning it until good vibration reduction effect is observed. In the future studies, a suitable algorithm can be used to automatically tune the $L$ value based on the real-time control effect.

Using Eq. (12) to guess an initial $L$ value requires the knowledge of the patch's intrinsic capacitance. The impedance of the patch glued on the structure is usually measured to obtain a capacitance value [15]. In fact this value is not the intrinsic capacitance of the patch since the measured impedance includes the contribution of the host structure. Here a different way is used to obtain this capacitance. The resonance frequency of the circuit is the upper boundary of the band gap created by the resonant shunts [30]. Therefore, the intrinsic capacitance in the experiments can be obtained by first estimating the upper boundary $\omega_{u p}$ of a gap when the $L$ value is given then using $C_{p}=1 / \omega_{u p}^{2} / L$. The estimated capacitance in the experiment is around $C_{p}=400 \mathrm{nF}$.

In numerical simulations, the intrinsic capacitance of the patch can be determined using

$$
C_{p}=\frac{\varepsilon_{3}^{\sigma} l_{p} b}{h_{p}}
$$

in which, $\varepsilon_{3}^{\sigma}$ is the permittivity at constant stress. The capacitance in simulations is $C_{p}=95.4 n F$.

Before studying the programmable dynamical behaviors of the metamaterial beam, the electro-mechanical coupling factor of the patches should be analyzed. There will be no control effect for the targeted mode if the patches are not coupled to it. The modal coupling factor (MCF) is used in this paper, it is estimated using [27]

$$
\alpha_{c f, i}=\frac{\omega_{o c, i}^{2}-\omega_{s c, i}^{2}}{\omega_{o c, i}^{2}},
$$

in which, $\omega_{s c, i}$ and $\omega_{o c, i}$ are the $i$ th resonance frequencies when the patches for control are $\mathrm{SC}$ and $\mathrm{OC}$, respectively.

The global MCF of all the patches to one particular mode is studied. The transfer functions between the excitation voltage $V$ and the transverse acceleration $a_{z}$ at the left end of the beam are measured when all the patches for control are SC and OC, 
respectively. The results are shown in Fig. 5(a). Fifteen flexural modes are observed from $100 \mathrm{~Hz}$ to $2000 \mathrm{~Hz}$ in Fig. 5(a), their resonance frequencies at SC and OC cases can be determined. Note that, a Bragg-type band gap is also observed in Fig. 5(a), it is caused by the periodic nature of the studied metamaterial beam. Using the determined resonance frequencies and Eq. (14), the global MCFs are obtained, as illustrate in Fig. 5(b). The corresponding MCFs of these modes in simulation are also shown in Fig. 5(c) as references. These studied modes are actually the 5th to 20th flexural modes of the metamaterial beam. Without making any confusion and for clarity, they are called the 1 st to 15 th modes in the discussion. Overall, it is observed that except the 15 th one there is no mode with near-zero global MCF, i.e., all the first 14 modes can be well controlled by the patches. It is also noticed that, the global MCFs for the modes above the Bragg-gap are relatively much smaller than those below the gap. This feature is related to the mode shapes of the metamaterial beam. The beam has a thick and thin part in each unit cell, the patches are glued on the thin parts. The shapes of the modes below the Bragg-gap have most of their nodal lines in the thick parts (see the mode shape of the 6th mode in $5(\mathrm{c})$ as an example), results in less charge neutralization on the electrodes of the patches, which means high coupling factor between the strain energy and electrical energy. In the contrary, the nodal lines of the mode shapes above the gap quite often occur in the thin parts, leading to significant charge neutralization on the terminals of the patches glued on these parts.

\subsection{Programmable dynamical behaviors}

In many situations, it is important to mitigate the vibration of particular modes of structures. The targeted modes could be different in different working conditions or environments. Therefore, it is very useful if the structure can be online programmed to always reduce the vibration of desired modes. The proposed programmable metamaterial in this paper just has such merit. To analyze the programmable behaviors of the metamaterial beam, 5 cases are studied with different control parameters, as summarized in Table 2. In the table, in order to compare the control parameters used in the simulation and experiment, the normalized $L$ and $R$ are used, which are

$$
L_{\text {norm }}=\omega_{s c}^{2} C_{p} L, R_{\text {norm }}=\omega_{s c} C_{p} R
$$

in which, $\omega_{s c}$ is the resonance frequency of the targeted mode at SC condition.

In each case, the designed control parameters are implemented in all the 9 digital circuits, the metamaterial beam is programmed to achieve different vibration reduction effects. In the cases 1, 2 and 3, three different $L$ values are used to target the 3rd, 6th and 8th modes, respectively. Dispersion curves in these three cases are illustrated in Fig. 6(a) and the numerical transfer functions are presented in Figs. 6(b). Dispersion analysis in Fig. 6(a) shows that locally resonant (LR) band gaps are created near the corresponding targeted modes. The simulated transfer functions in Fig. 6(b) further confirm the existence of the three band gaps, the targeted modes are totally removed. The experimental results are shown in Fig. 6(c), these results illustrate that the designed metamaterial can be programmed to create band gaps at desired frequencies to remove targeted modes just as in the simulation. 


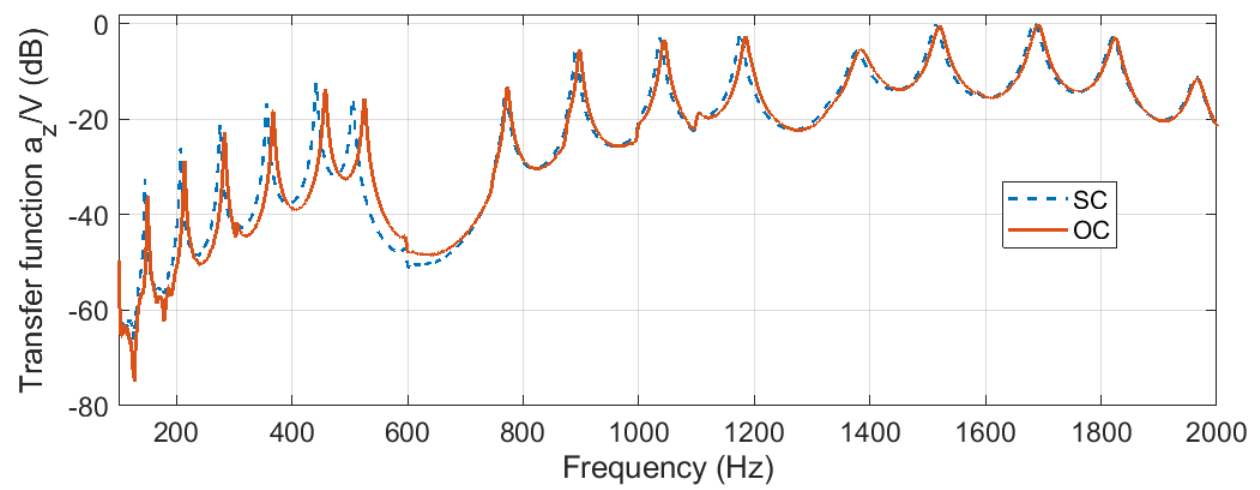

(a) Measured transfer fucntion

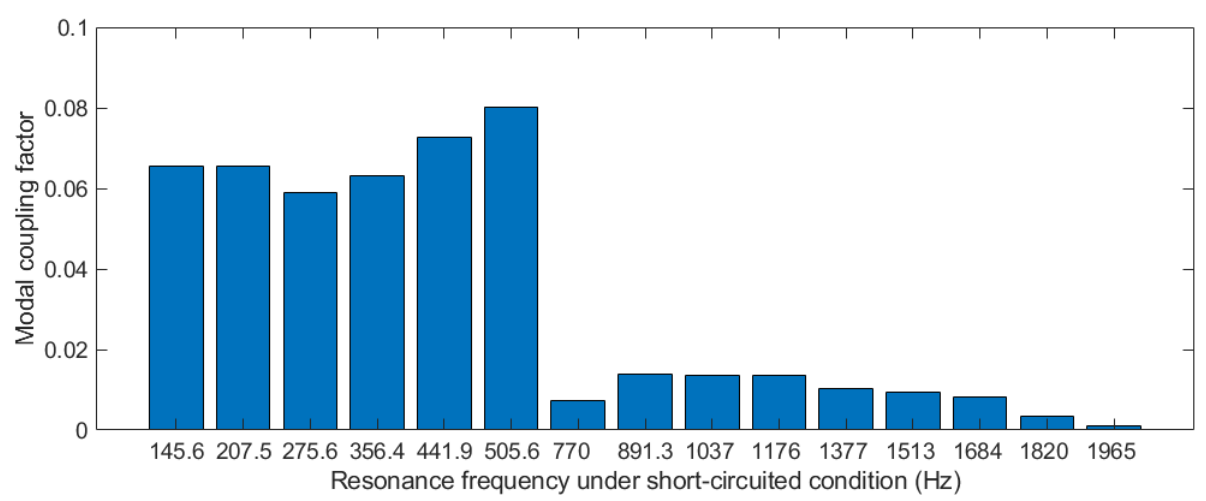

(b) Measured global MCFs

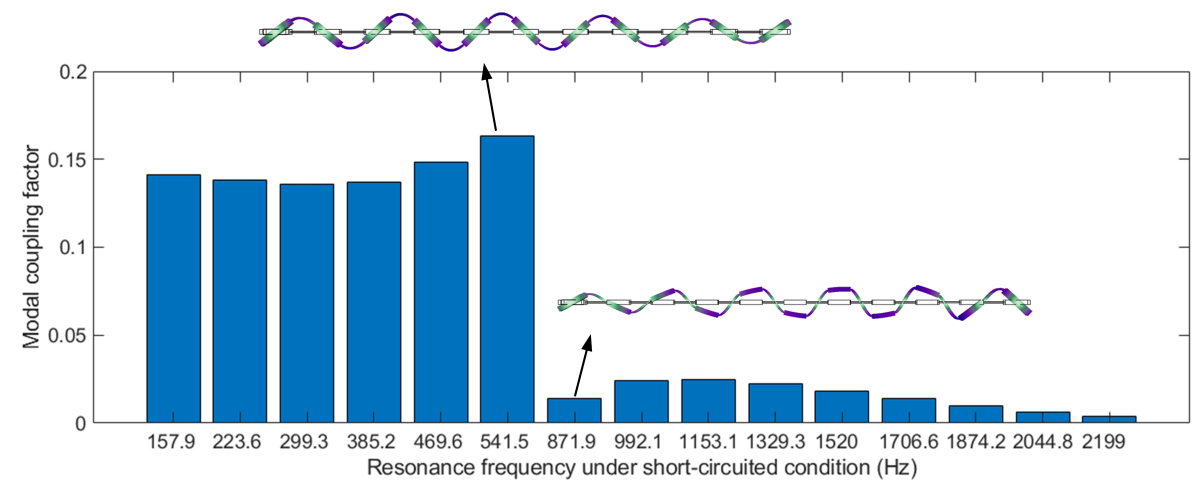

(c) Simulated global MCFs

Figure 5: (a) Measured transfer functions between the excitation voltage $V$ and the measured transverse acceleration $a_{z}$ when all the patches for control are SC and OC, respectively. Global MCFs obtained in the (a) experiment and (b) simulation. In Fig. 5(c), the mode shapes for the 6th and 7th modes are also shown. 

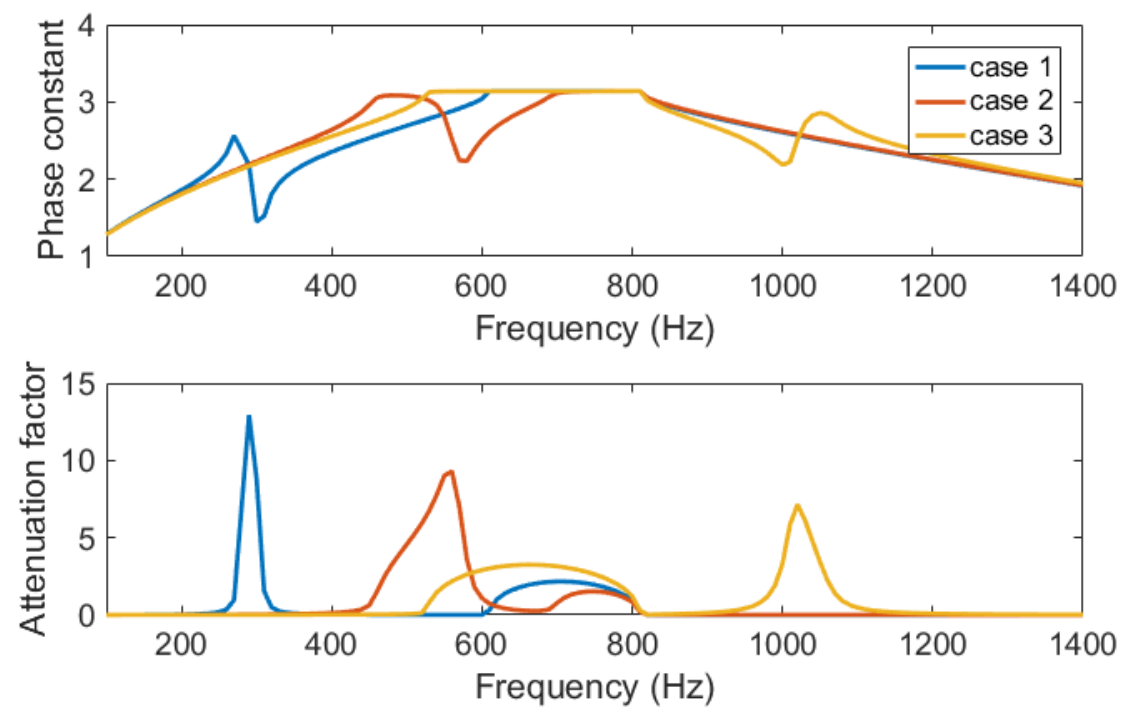

(a) Dispersion curves

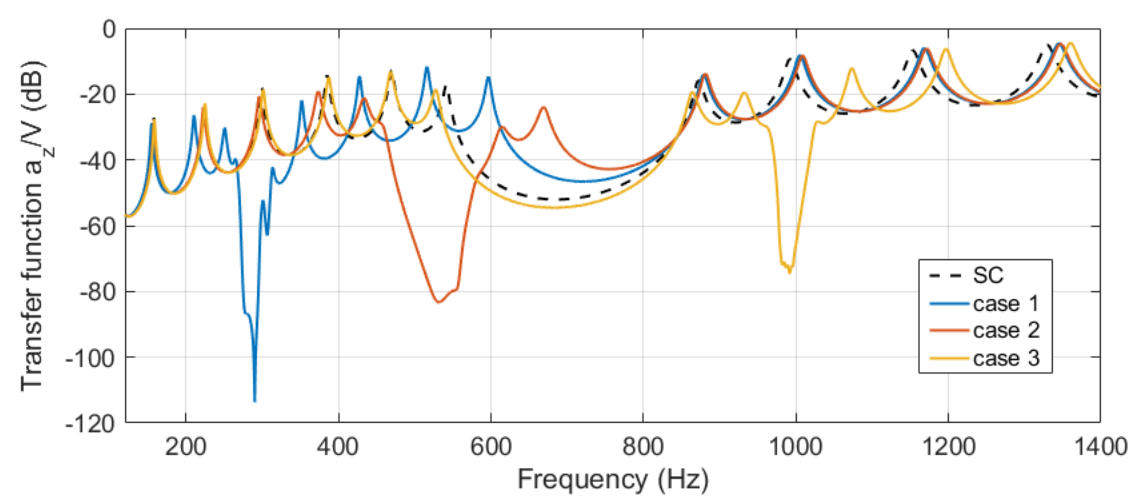

(b) Simulation

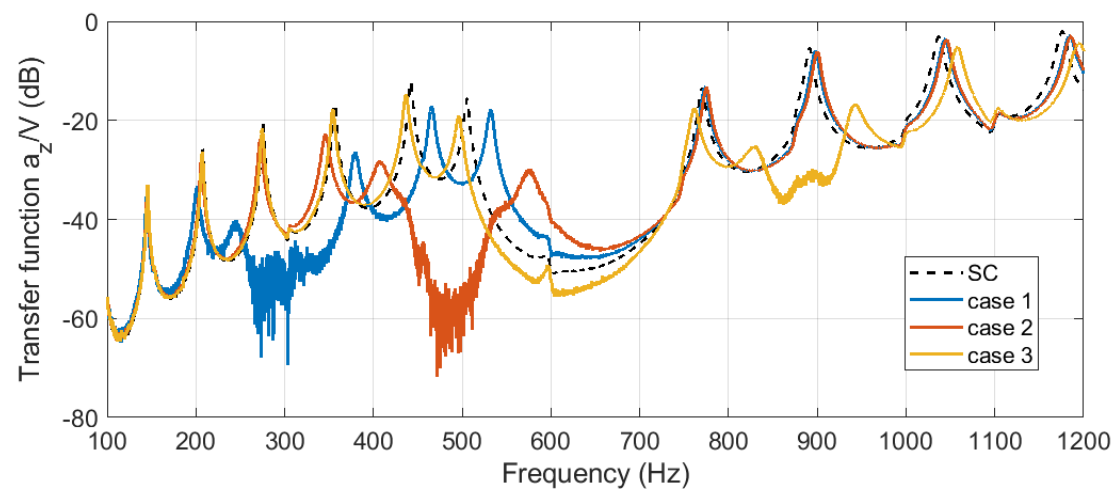

(c) Experiment

Figure 6: Tuning the band gaps to target different modes. (a) dispersion curves, (b) simulated transfer functions and (c) measured transfer functions. 
Table 2: Normalized $L$ and $R$ values used in the studied cases.

\begin{tabular}{ccccc}
\hline & case & frequency $(\mathrm{Hz}) /$ mode order & $L_{\text {norm }}$ & $R_{\text {norm }}$ \\
\hline \multirow{5}{*}{ simulation } & 1 & $299 /$ rd & 0.0179 & 0.0023 \\
& 2 & $541 / 6$ th & 0.0165 & 0.0041 \\
& 3 & $992 / 8$ th & 0.0178 & 0.0038 \\
& 4 & $541 / 6$ th & 0.0165 & 0.0206 \\
experiment & 5 & $541 / 6$ th & 0.0165 & 0.1032 \\
& 1 & $276 / 3 \mathrm{rd}$ & 0.0189 & 0.0022 \\
& 2 & $506 / 6$ th & 0.0194 & 0.004 \\
& 4 & $891 / 8$ th & 0.0188 & 0.0036 \\
& 5 & $506 / 6$ th & 0.0194 & 0.0202 \\
\end{tabular}

From case 1 to case 3, the gaps caused by resonant behaviors are narrow, they are not wide enough to cover the desired frequency ranges in some circumstances. To broaden the effective region of the gap, one method is to combine different types of gaps to form a hybrid gap zone. There are simultaneously two different gaps in the designed metamaterial beam, one LR gap and one Bragg-gap, as shown in Fig. 6. The LR gap can be shifted to the lower boundary of the Bragg-gap in order to form a wider hybrid gap, as shown in Fig. 7. In this design, the $L$ parameter in the control law is first tuned to move the LR gap (case 2). After determining the $L$ value, the $R$ parameter is further tuned to enhance the damping introduced by the circuits (cases 4 and 5) to further broaden the effective zone of the gap. It can be observed that, in case 5 the LR gap and Bragg-gap are well combined (see FIg. 7(a)). This wide gap region is also observed in the experiment, a hybrid gap from nearly $360 \mathrm{~Hz}$ to $770 \mathrm{~Hz}$ is obtained, the modes within this region are almost removed (see Fig. 7(c)). Besides, amplitudes of the three modes (1st to 3rd) before the hybrid gap region are also reduced by around $10 \mathrm{~dB}$ or more.

The above method can only be used to broaden the effective zone of vibration reduction near the Bragg-gap. Another way to program the metamaterial to realize vibration reduction in a wide and flexible frequency band is to program the digital circuits to have different but successive resonance frequencies, therefore to realize a "rainbow trap" [31]. Without loss any generality, the resonance frequencies of the first nine modes are chosen, one digital circuit is programmed to resonate near one of these frequencies.

In this design, it must be avoid to use a set of patch to control the mode with very low MCF. Therefore, the MCF of each patch set to one particular mode is measured first. To measure the MCFs of a patch set, it is kept OC and the others are SC, the resonance frequencies in this situation are determined according to the measured transfer function, then the MCFs are estimated using Eq. (14). Results are shown in Fig. 8. There are 9 bars at each frequency, which represent the MCFs of the 9 patch sets used for control. There is no need in using the best coupled patches for the corresponding mode, the more important aim is to guarantee the control effect of all the 9 modes. It can be observed from Fig. 8 that the MCFs for the last 3 modes are relatively lower, the controllers for them are chosen first. The 1st controller is used to control the 7 th mode, 

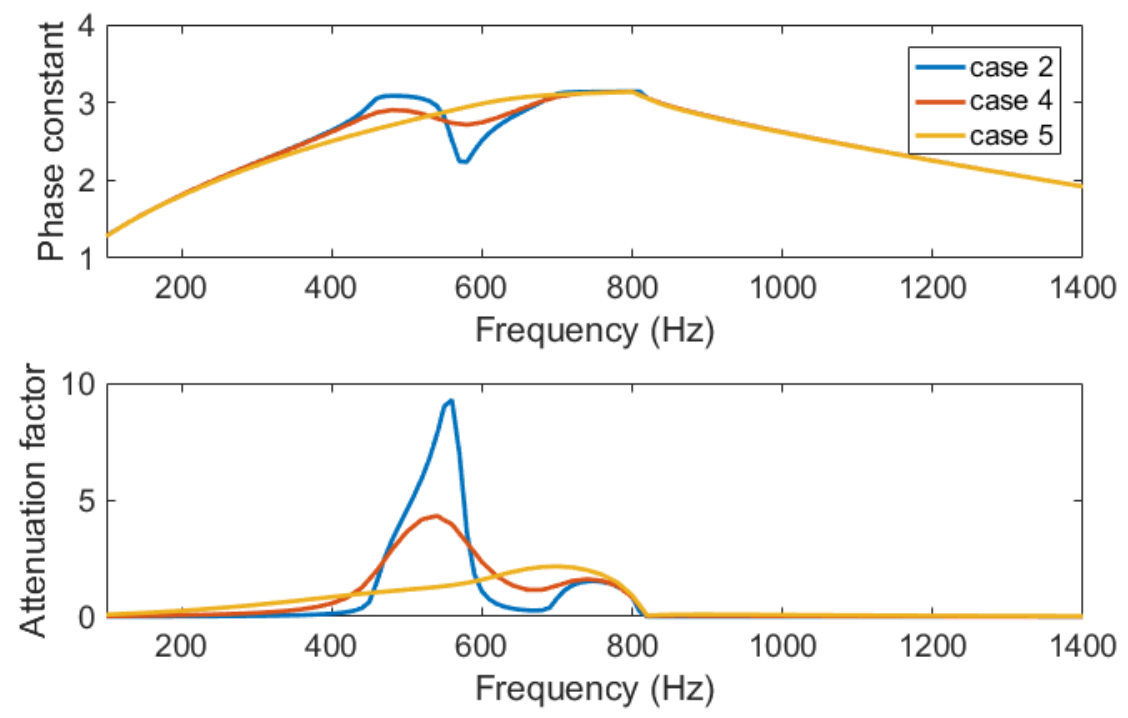

(a) Dispersion curves

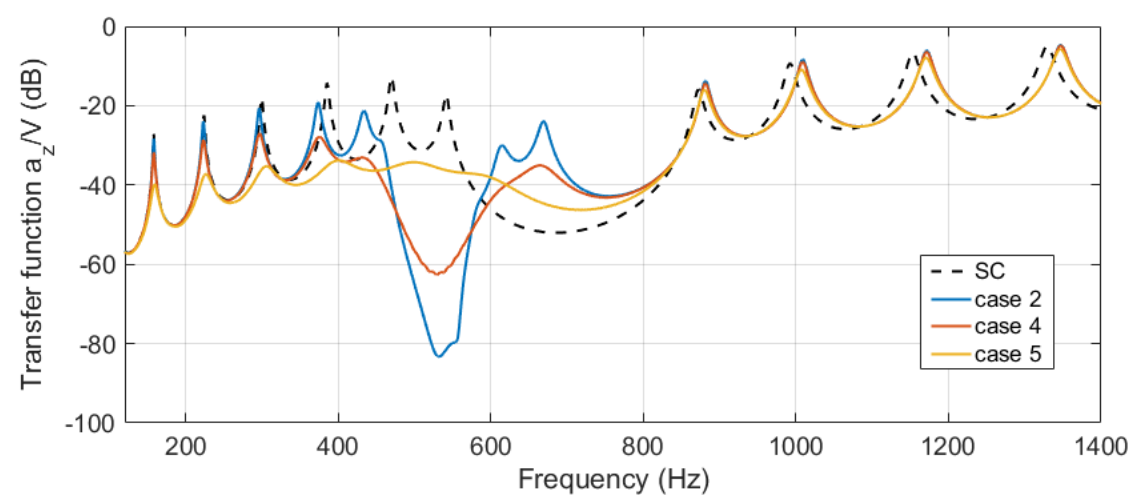

(b) Simulation

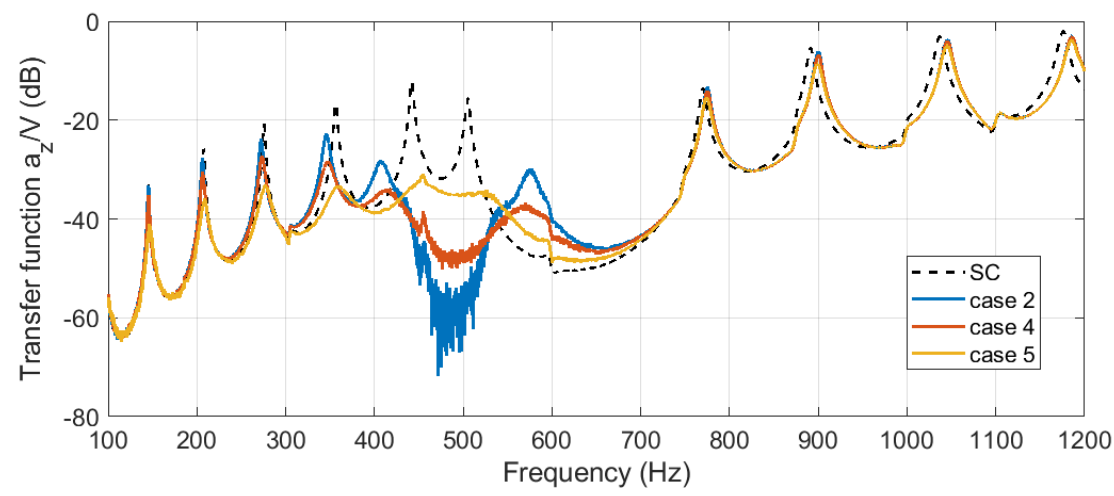

(c) Experiment

Figure 7: Tuning the damping to enlarge the effective zone of the LR gap. (a) dispersion curves, (b) simulated transfer functions and (c) measured transfer functions. 


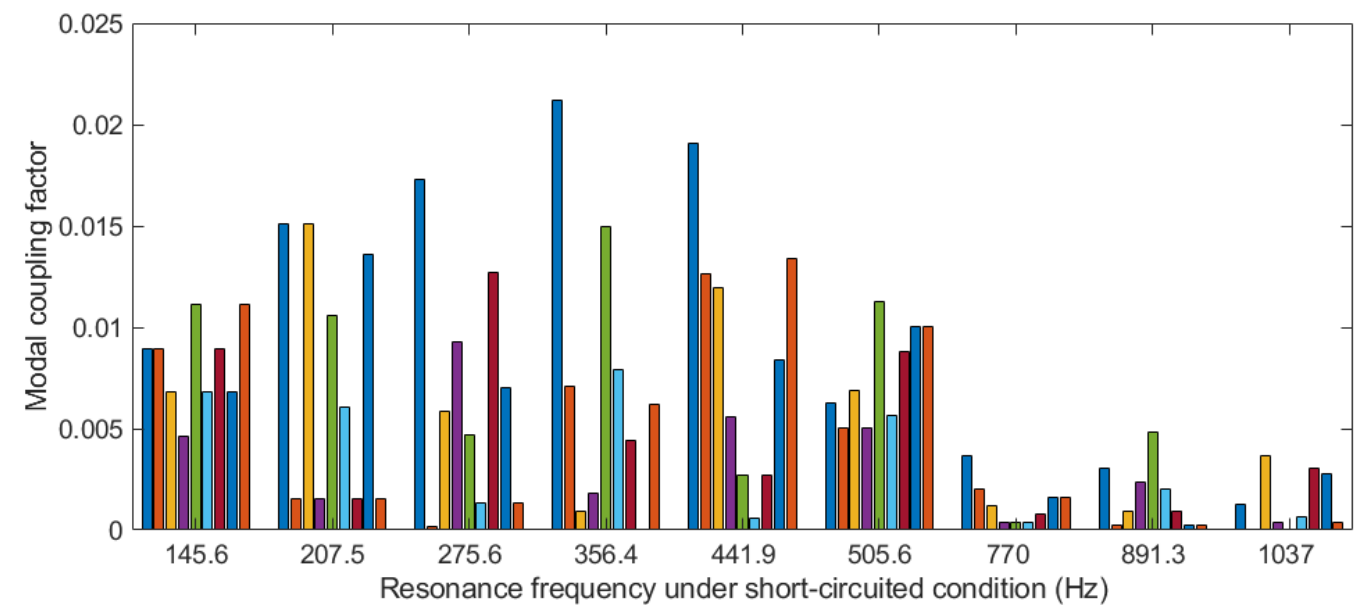

Figure 8: Measured modal coupling factor for each patch set. There are 9 bars at each frequency, which represent the modal coupling factors of the 9 patch sets used for control.

and the 5th and 3rd are respectively for the 8th and 9th modes. The controllers for the other 6 modes are quite flexible. Table 3 summarizes the mode-controller relationships and the final parameters used in the corresponding control law. The parameters for the 9 controllers are manually tuned one by one as mentioned before. Figure 9 shows the vibration reduction effect in the simulation and experiment. It can be observed that the numerical and experimental results have good coincidence in terms of the control effect. In the experiment, the amplitudes of all the targeted modes are reduced by almost $15 \mathrm{~dB}$ or more. These results demonstrate the impressive abilities of the programmable metamaterial in the application of vibration control within a desired wide frequency band.

\section{Conclusions}

This paper studies a new type of smart metamaterial consisting of piezoelectric materials and digital synthetic impedance circuits (digital circuits), its programmable dynamical behaviors in terms of vibration reduction are studied through experiments and numerical simulations. The digital circuits are used to simulate behaviors of resonant shunts therefore to program the equivalent bending stiffness and damping of the metamaterial beam. Different design methods are used to customize the dynamical behaviors of the metamaterial beam for the purpose of vibration reduction. The metamaterial beam is programmed to have LR band gaps at desired locations to remove targeted modes when all the circuits are controlled by the same law. It can also be programmed to have a LR gap near a Bragg-gap therefore to obtain a larger hybrid gap. Tuning the damping introduced by the digital circuits can further enlarge the effective region of the hybrid gap. The metamaterial beam can be programmed to realize a "rainbow trap" therefore to mitigate vibration in a large frequency band, which covers 9 modes in our experimental study. The amplitudes of all the 9 targeted modes are reduced by almost $15 \mathrm{~dB}$ or more. The results in this paper demonstrate that the proposed smart metamaterial can be flexibly programmed to reduce vibration at desired frequencies or within a desired large 
Table 3: The targeted mode-controller relationships and the normalized $L$ and $R$ values used in the corresponding digital circuits.

\begin{tabular}{ccccc}
\hline & frequency $(\mathrm{Hz}) /$ mode order & controller & $L_{\text {norm }}$ & $R_{\text {norm }}$ \\
\hline $169 /$ st & 9 & 0.0171 & 0.0177 \\
$239 / 2$ nd & 8 & 0.0166 & 0.0228 \\
$319 / 3$ rd & 4 & 0.0167 & 0.0243 \\
$411 / 4$ th & 6 & 0.0173 & 0.0196 \\
simulation & $503 / 5$ th & 2 & 0.0192 & 0.0192 \\
& $584 / 6$ th & 7 & 0.0186 & 0.0223 \\
$878 / 7$ th & 1 & 0.018 & 0.0084 \\
& $1004 / 8$ th & 5 & 0.017 & 0.0115 \\
& $1167 / 9$ th & 3 & 0.0177 & 0.0134 \\
\hline $146 / 1$ st & 9 & 0.0244 & 0.0006 \\
$208 / 2$ nd & 8 & 0.0195 & 0.0066 \\
$276 / 3$ rd & 4 & 0.0177 & 0.0066 \\
$356 / 4$ th & 6 & 0.0182 & 0.0071 \\
experiment & $442 / 5$ th & 2 & 0.017 & 0.0124 \\
& $506 / 6$ th & 7 & 0.0177 & 0.0121 \\
& $770 / 7$ th & 1 & 0.0185 & 0.0077 \\
$891 / 8$ th & 5 & 0.0177 & 0.0107 \\
& $1037 / 9$ th & 3 & 0.0175 & 0.0104 \\
\hline
\end{tabular}




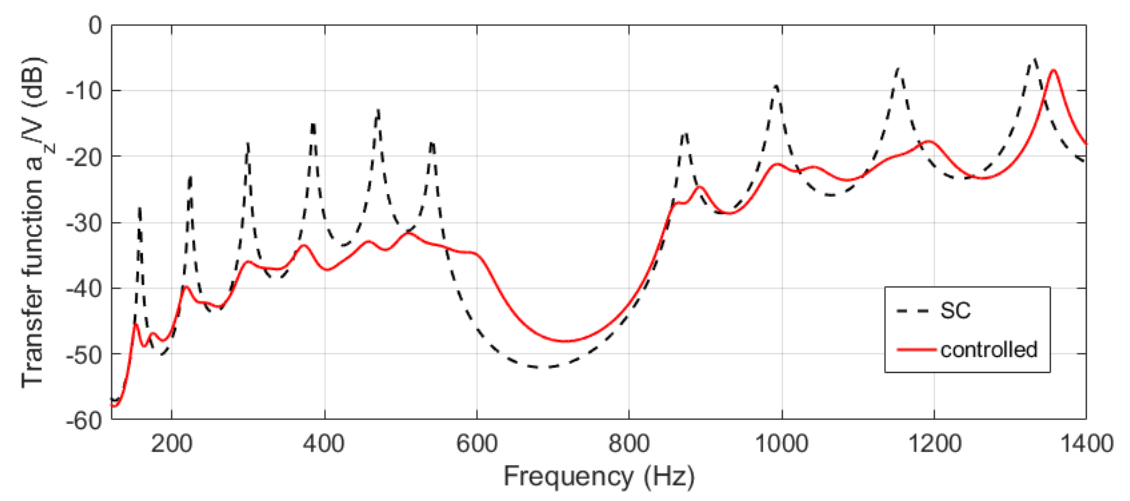

(a) Simulation

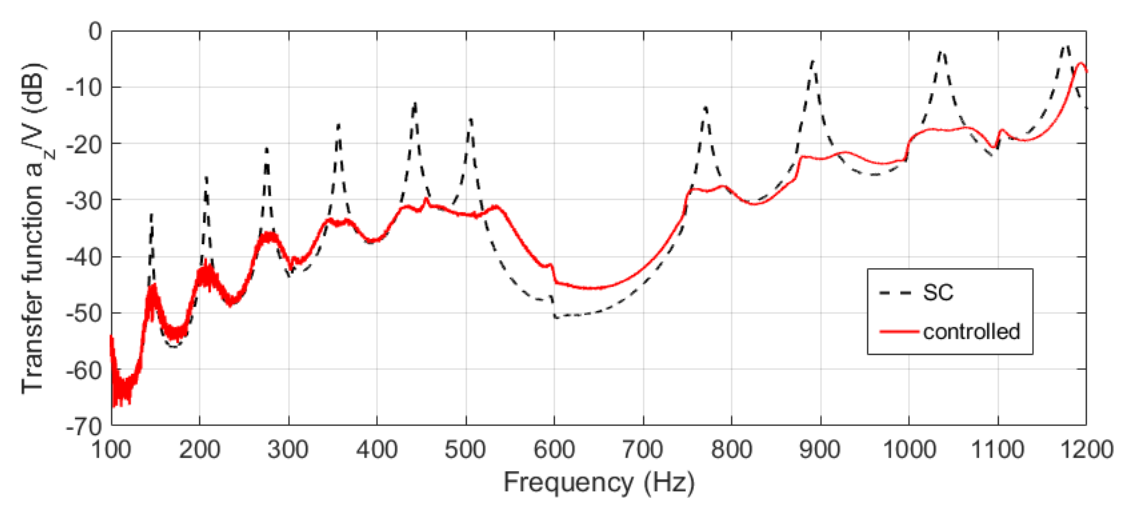

(b) Experiment

Figure 9: Broadband vibration reduction effect. 
frequency band. The proposed conception of programmable metamaterials can be naturally extended to other types of structures, such as plates and shells, they are promising components in the future adaptive structures.

\section{Acknowledgement}

This work was supported by the EUR EIPHI project (contract ANR-17-EURE-0002) and Bourgogne Franche-Comté Region.

\section{Appendix A. Materials parameters}

The Young's modulus and density of aluminum are $70 \mathrm{GPa}$ and $2700 \mathrm{~kg} / \mathrm{m}^{3}$, respectively. The parameters of the PIC 151 are listed in table A.4.

Table A.4: Material parameters of PIC 151.

\begin{tabular}{ccc}
\hline Symbol & Value & Property \\
\hline$S_{11}^{E}=S_{22}^{E}, S_{33}^{E}$ & $1.683 \times 10^{-11}, 1.9 \times 10^{-11}\left(\mathrm{~Pa}^{-1}\right)$ & Compliance matrix under \\
$S_{12}^{E}, S_{13}^{E}=S_{23}^{E}$ & $-5.656 \times 10^{-12},-7.107 \times 10^{-12}\left(\mathrm{~Pa}^{-1}\right)$ & constant electric field \\
$S_{44}^{E}=S_{55}^{E}, S_{66}^{E}$ & $5.096 \times 10^{-11}, 4.497 \times 10^{-11}\left(\mathrm{~Pa}^{-1}\right)$ & \\
\hline$d_{31}=d_{32}$ & $-2.14 \times 10^{-10}(\mathrm{C} / \mathrm{N})$ & Piezoelectric matrix \\
$d_{33}$ & $4.23 \times 10^{-10}(\mathrm{C} / \mathrm{N})$ & \\
$d_{24}=d_{15}$ & $6.1 \times 10^{-10}(\mathrm{C} / \mathrm{N})$ & Density \\
$\rho$ & $7760\left(\mathrm{~kg} / \mathrm{m}^{3}\right)$ & Dielectric permittivity \\
$\varepsilon_{1}^{\sigma}=\varepsilon_{2}^{\sigma}, \varepsilon_{3}^{\sigma}$ & $1936 \varepsilon_{0}, 2109 \varepsilon_{0}$ & under constant stress \\
\hline
\end{tabular}

\section{References}

\section{References}

[1] M. Collet, M. Ouisse, and F. Tateo. Adaptive metacomposites for vibroacoustic control applications. IEEE Sensors Journal, 14(7):2145-2152, 2014.

[2] F Casadei, M Ruzzene, Lorenzo Dozio, and KA Cunefare. Broadband vibration control through periodic arrays of resonant shunts: experimental investigation on plates. Smart materials and structures, 19(1):015002, 2009.

[3] Hao Zhang, Jihong Wen, Yong Xiao, Gang Wang, and Xisen Wen. Sound transmission loss of metamaterial thin plates with periodic subwavelength arrays of shunted piezoelectric patches. Journal of Sound and Vibration, 343:104-120, 2015. 
[4] Manuel Collet, Morvan Ouisse, Mohammed Ichchou, and Roger Ohayon. Semi-active optimization of 2d wave's dispersion into shunted piezocomposite systems for controlling acoustic interaction. In ASME 2011 Conference on Smart Materials, Adaptive Structures and Intelligent Systems, pages 79-87. American Society of Mechanical Engineers Digital Collection, 2012.

[5] Z. Liu, X. Zhang, Y. Mao, Y. Y. Zhu, Z. Yang, C. T. Chan, and S. Ping. Locally resonant sonic materials. Science, 289(5485):1734-1736, 2000.

[6] R. L. Forward. Electronic damping of vibrations in optical structures. Appl Opt, 18(5):690-697, 1979.

[7] N. W. Hagood and A. V. Flotow. Damping of structural vibrations with piezoelectric materials and passive electrical networks. Journal of Sound Vibration, 146(2):243268, 1991.

[8] O. Thorp, M. Ruzzene, and A. Baz. Attenuation and localization of wave propagation in rods with periodic shunted piezoelectric patches. In Spies International Symposium on Smart Structures Materials, 2001.

[9] A. Bergamini, T. Delpero, L. Simoni, L. Lillo, M. Ruzzene, and P. Ermanni. Phononic crystal with adaptive connectivity. Advanced Materials, 26(9):1343-1347, 2014.

[10] W. Gang and S. Chen. Large low-frequency vibration attenuation induced by arrays of piezoelectric patches shunted with amplifier-resonator feedback circuits. Smart Materials Structures, 25(1):015004, 2016.

[11] S. Chen, W. Gang, J. Wen, and X. Wen. Wave propagation and attenuation in plates with periodic arrays of shunted piezo-patches. Journal of Sound Vibration, 332(6):1520-1532, 2013.

[12] L Airoldi and M Ruzzene. Design of tunable acoustic metamaterials through periodic arrays of resonant shunted piezos. New Journal of Physics, 13(11):113010, 2011.

[13] R. L. Forward. Electromechanical transducer-coupled mechanical structure with negative capacitance compensation circuit. US Patent Specification, 4(158):787, 1979.

[14] B. S. Beck, K. A. Cunefare, and M. Collet. The power output and efficiency of a negative capacitance shunt for vibration control of a flexural system. Smart Materials Structures, 22(6):065009, 2013.

[15] M. Berardengo, O. Thomas, C. Giraudaudine, and S. Manzoni. Improved resistive shunt by means of negative capacitance: new circuit, performances and multi-mode control. Smart Material Structures, 25(7):075033, 2016.

[16] Y. Fan, M. Collet, M. Ichchou, L. Li, O. Bareille, and Z. Dimitrijevic. A wave-based design of semi-active piezoelectric composites for broadband vibration control. Smart Materials and Structures, 25(5):055032, 2016. 
[17] R. Zhu, Y.Y. Chen, M.V. Barnhart, G.K. Hu, C.T. Sun, and G.L. Huang. Experimental study of an adaptive elastic metamaterial controlled by electric circuits. Applied Physics Letters, 108(1):011905, 2016.

[18] K. Yi, M. Collet, M. Ichchou, and L. Li. Flexural waves focusing through shunted piezoelectric patches. Smart Materials and Structures, 25(7):075007, 2016.

[19] G. Trainiti, Y. Xia, J. Marconi, A. Erturk, and M. Ruzzene. Time-periodic stiffness modulation in elastic metamaterials for selective wave filtering: theory and experimental investigations. 2018.

[20] A. J. Fleming and S. O. Reza Moheimani. Optimal impedance design for piezoelectric vibration control. In IEEE Conference on Decision and Control, 2013.

[21] A. J. Fleming and S. O. R. Moheimani. Control orientated synthesis of highperformance piezoelectric shunt impedances for structural vibration control. IEEE Transactions on Control Systems Technology, 13(1):98-112, 2004.

[22] Andrew J. Fleming. Improved current and charge amplifiers for driving piezoelectric loads. Proceedings of SPIE - The International Society for Optical Engineering, 15(2):77-92, 2003.

[23] A. J. Fleming, S. Behrens, and S. O. R. Moheimani. Synthetic impedance for implementation of piezoelectric shunt-damping circuits. Electronics Letters, 36(18):15251526, 2002.

[24] Andrew J. Fleming, Sam Behrens, and S. O. Reza Moheimani. Optimization and implementation of multimode piezoelectric shunt damping systems. IEEE/ASME Transactions on Mechatronics, 7(1):87-94, 2002.

[25] G. Matten, M. Collet, S. Cogan, and E. Sadoulet-Reboul. Synthetic impedance for adaptive piezoelectric metacomposite. Procedia Technology, 15:84-89, 2014.

[26] J. Nečásek, J. Václavík, and P. Marton. Digital synthetic impedance for application in vibration damping. Review of Scientific Instruments, 87(2):024704, 2016.

[27] C. Sugino, M. Ruzzene, and A. Erturk. Design and analysis of piezoelectric metamaterial beams with synthetic impedance shunt circuits. IEEE/ASME Transactions on Mechatronics, 23(5):2144-2155, 2018.

[28] M. Collet, M. Ouisse, M. Ruzzene, and M. N. Ichchou. Floquet-bloch decomposition for the computation of dispersion of two-dimensional periodic, damped mechanical systems. International Journal of Solids Structures, 48(20):2837-2848, 2011.

[29] J. M. Mencik and M. N. Ichchou. Multi-mode propagation and diffusion in structures through finite elements. European Journal of Mechanics, 24(5):877-898, 2005.

[30] C. Sugino, S. Leadenham, M. Ruzzene, and A. Erturk. An investigation of electroelastic bandgap formation in locally resonant piezoelectric metastructures. Smart Materials Structures, 26(5):055029, 2017. 
[31] D. Cardella, P. Celli, and S. Gonella. Manipulating waves by distilling frequencies: a tunable shunt-enabled rainbow trap. Smart Materials and Structures, 25(8):085017, 2016. 